\title{
Multicentre randomised controlled trial of nursing intervention for breathlessness in patients with lung cancer
}

\author{
Mary Bredin, Jessica Corner, Meinir Krishnasamy, Hilary Plant, Chris Bailey, Roger A’Hern
}

\begin{abstract}
Objective To evaluate the effectiveness of nursing intervention for breathlessness in patients with lung cancer.

Design Patients diagnosed with lung cancer participated in a multicentre randomised controlled trial where they either attended a nursing clinic offering intervention for their breathlessness or received best supportive care. The intervention consisted of a range of strategies combining breathing control, activity pacing, relaxation techniques, and psychosocial support. Best supportive care involved receiving standard management and treatment available for breathlessness, and breathing assessments. Participants completed a range of self assessment questionnaires at baseline, 4 weeks, and 8 weeks.
\end{abstract}

Setting Nursing clinics within 6 hospital settings in the United Kingdom.

Participants 119 patients diagnosed with small cell or non-small cell lung cancer or with mesothelioma who had completed first line treatment for their disease and reported breathlessness.

Outcome measures Visual analogue scales measuring distress due to breathlessness, breathlessness at best and worst, WHO performance status scale, hospital anxiety and depression scale, and Rotterdam symptom checklist.

Results The intervention group improved significantly at 8 weeks in 5 of the 11 items assessed: breathlessness at best, WHO performance status, levels of depression, and two Rotterdam symptom checklist measures (physical symptom distress and breathlessness) and showed slight improvement in 3 of the remaining 6 items.

Conclusion Most patients who completed the study had a poor prognosis, and breathlessness was typically a symptom of their deteriorating condition. Patients who attended nursing clinics and received the breathlessness intervention experienced improvements in breathlessness, performance status, and physical and emotional states relative to control patients.

\section{Introduction}

Breathlessness is increasingly recognised as not simply a symptom of disordered breathing but also a complex interplay of physical, psychological, emotional, and functional factors. ${ }^{1}$ Between $10 \%$ and $15 \%$ of patients with lung cancer have breathlessness at diagnosis, and $65 \%$ will have the symptom at some point during their illness. ${ }^{2}$ Alongside cough, it is the symptom most frequently reported by patients with lung cancer. ${ }^{3}$ The subjective experience of breathlessness may not be directly related to the extent of the disease. Factors such as anxiety can play an important part in exacerbating the symptom, and this is particularly evident in the context of an imminently life threatening illness such as lung cancer. ${ }^{4}$

Pharmacological and non-pharmacological interventions for breathlessness have not been evaluated. Treatment has focused on drainage of pleural effusions and on pharmacological interventions aimed at reducing perception of the symptom-but evidence suggests that breathlessness remains unrelieved despite the use of recognised palliative interventions. ${ }^{5}$

Corner and colleagues set out to identify and evaluate nursing strategies for managing breathlessness and adopted an integrated approach that emphasised the importance of not separating psychological and physical aspects of the symptom. ${ }^{4}$ They developed a therapeutic intervention that aimed to increase fitness and tolerance of restricted lung function and reduce functional disability while acknowledging the meaning of breathlessness in the context of life threatening illness. Results from a small randomised controlled study of patients attending a breathing clinic indicated the potential value of the intervention, particularly in the areas of distress caused by breathlessness, functional ability, and ability to perform activities of daily living. ${ }^{6}$ This was a single institution study of 34 patients; a multicentre study was organised to evaluate the effect of the intervention on a larger, more diverse sample and to establish the feasibility of integrating the new approach in a range of treatment centres.

\section{Methods}

\section{Study design}

This multicentre study was coordinated from the Macmillan Practice Development Unit at the Centre for Cancer and Palliative Care Studies, Institute of Cancer Research, London. Patients diagnosed with small cell lung cancer, non-small cell lung cancer, or mesothelioma who had completed treatment and reported breathlessness were invited to take part in the study. Entry criteria for the study defined shortness of breath as a reported change in breathing or a degree of breathlessness as perceived by the patient and reported as a problem that caused distress.

In each of the participating centres, once a patient from one of the participating centres had consented to take part in the trial, a telephone call was made to the Institute of Cancer Research's clinical trials office, which was responsible for independent randomisation to either intervention or control groups. The trials office informed the participating centre which group the patient had been assigned to. The patient was then asked to confirm whether he or she remained happy to participate in the study. Patients in the control group

\author{
Editorial \\ by Delamothe et al \\ Centre for Cancer \\ and Palliative Care \\ Studies, Macmillan \\ Practice \\ Development Unit, \\ Institute of Cancer \\ Research, Royal \\ Marsden NHS \\ Trust, London SW3 \\ 6JJ \\ Mary Bredin, \\ Macmillan research \\ practitioner \\ Meinir \\ Krishnasamy, \\ Macmillan research \\ coordinator \\ Centre for Cancer \\ and Palliative Care \\ Studies, Institute of \\ Cancer Research \\ Jessica Corner, \\ professor of cancer \\ nursing \\ Hilary Plant, \\ research practitioner \\ Chris Bailey, \\ research practitioner \\ Department of \\ Computing and \\ Information, Royal \\ Marsden NHS Trust \\ Roger A'Hern, \\ statistician \\ Correspondence to: \\ Jessica Corner \\ jessica@icr.ac.uk
}


- Detailed assessment of breathlessness and factors that ameliorate or exacerbate it

- Advice and support for patients and their families

- Exploration of the meaning of breathlessness, their disease, and feelings about the future

- Training in breathing control techniques, progressive muscle relaxation, and distraction exercises

- Goal setting to complement breathing and relaxation techniques, to help in the management of functional and social activities, and to support the development and adoption of coping strategies

- Early recognition of problems warranting pharmacological or medical intervention
Intervention carried out by specialist nurses on ways of managing breathlessness

\section{Recruitment of centres}

Six hospital centres from around the United Kingdom volunteered to join the study. Each centre was granted ethical approval from its local research ethics committee. Informed written consent was obtained from patients, who were also aware of their ability to withdraw from the study at any time. Supervision and implementation of the trial involved regular telephone contact and visits to each centre and meetings of all the participating centres at six month intervals to audit their intervention method. Steps were taken throughout the study to ensure the uniformity of the intervention across centres: all nurses taking part were taught the intervention in the same way, using a practice guideline, and they all completed a checklist at the end of each intervention session indicating the strategies they had used with patients (these were used to monitor correct delivery of the intervention).

were given standard care and also had their breathlessness and its effects on life monitored; patients in the intervention group attended a nursing clinic. In the nursing clinics, patients received a package of interventions tailored to individual patients (box) aimed at helping them to cope with breathlessness and maximise their existing lung function. Many of these strategies are commonly used in settings for patients with chronic lung diseases but are not routinely used with lung cancer patients. Best supportive care was defined as the standard management and treatment for breathlessness available to patients within each centre. This included pharmacological and palliative treatments and treatment of associated problems such as anxiety and depression. All patients taking part had access to all routinely available supportive care.

Patients in the intervention group were invited to attend the nursing clinic once a week for up to eight weeks (and for not less than three weeks) (see figure). Data were collected from both groups at weeks 1,4 , and 8. An independent data monitoring committee was set up to advise on the conduct of the study.

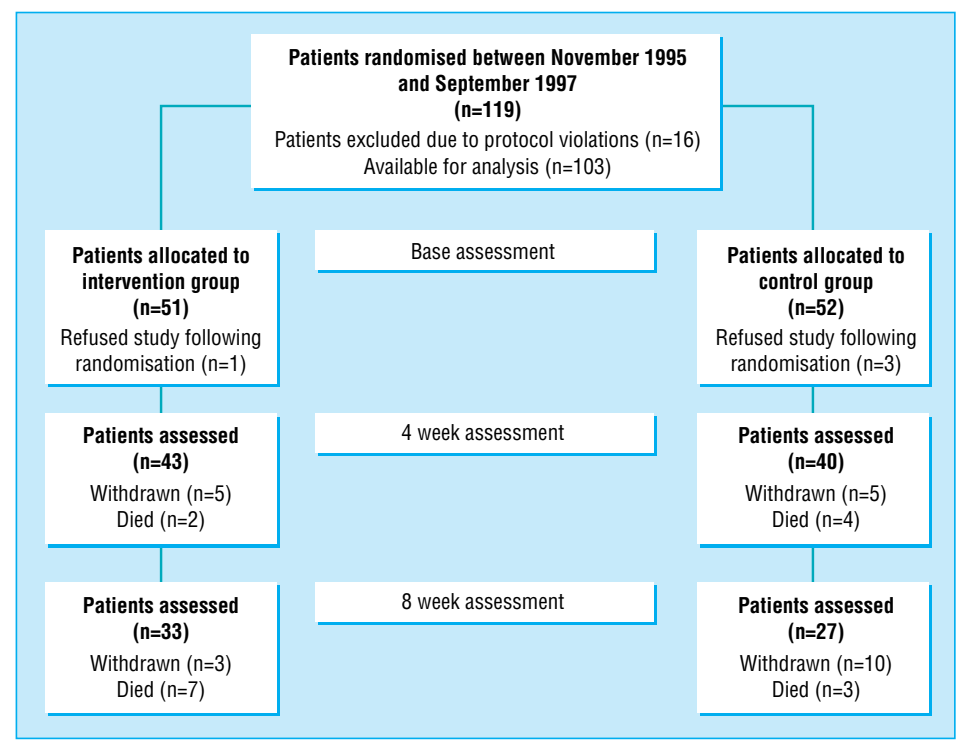

Design of study

\section{Outcome measures} assess the effects of the intervention. Patients' subjective experience of breathlessness was assessed with visual analogue scales measuring breathlessness at worst and at best and distress due to breathlessness. The primary outcome measure was distress due to breathlessness. Other measures included the WHO performance status scale, ${ }^{7}$ the hospital anxiety and depression scale, ${ }^{8}$ and the Rotterdam symptom checklist. $^{9}$

\section{Statistical methods}

Data from the research interviews and assessment instruments were entered onto EXCEL and SPSS-PC. As the data were not normally distributed, descriptive statistics and the non-parametric Mann-Whitney test were used in the analysis. The intended accrual was 150 patients to detect a difference in the proportion of patients who showed an improvement over 8 weeks, corresponding to $10 \%$ showing an improvement in one group and $30 \%$ in the other, or 25\% in one group and $50 \%$ in the other (approximate $90 \%$ power, $5 \%$ two sided significance level). In the final sample of 100 patients the power would be $70-75 \%$ but would have been increased as comparisons were not based on binomial outcomes but reflected the actual size of the changes.

At the outset of the study the principal time point chosen for analysis was from baseline to 8 weeks; we assumed that this was when the intervention would show its maximum impact. ${ }^{6}$ Patients who withdrew from the study for any reason other than that they change score that was one more (that is, worse) than the maximum of the patients who did not withdraw. Similarly, any patient who withdrew because he or she reported being too well to continue was given a score which was one less than the minimum score of the patients who did not withdraw. This method of treating withdrawals is recommended by Gould, ${ }^{10}$ who ranked patients who withdraw for reasons other than an improvement in their condition below patients who did not withdraw, and ranked those who withdrew because they improved above those who did not withdraw. No correction was made for multiple significance testing, although a very low critical significance level
Several self completed outcome measures were used to reported being too well to continue were given a 
Table 1 Baseline characteristics of 103 patients with lung cancer. Values are number of patients unless otherwise indicated

\begin{tabular}{lcc} 
& $\begin{array}{c}\text { Intervention } \\
\text { group }(\mathbf{n = 5 1 )}\end{array}$ & $\begin{array}{c}\text { Control group } \\
(\mathbf{n = 5 2})^{\star}\end{array}$ \\
\hline Mean (range) age (years) & $68(41-82)$ & $67(41-83)$ \\
\hline No (\%) men & $41(80)$ & $35(67)$ \\
\hline Diagnosis: & 8 & 6 \\
\hline Mesothelioma & 32 & 32 \\
\hline Non-small cell lung cancer & 11 & 13 \\
\hline Small cell lung cancer & 14 & 10 \\
\hline Metastatic disease & & \\
\hline Treatment: & 5 & 11 \\
\hline Surgery & 35 & 39 \\
\hline Radiotherapy & 15 & 15 \\
\hline Chemotherapy & & \\
\hline
\end{tabular}

${ }^{\star}$ Diagnosis not confirmed in one patient.

would have to be used if all the endpoints were considered to be independent. We did not use an adjustment because many of the outcomes are related and a multivariate non-parametric statistical adjustment for non-independent endpoints does not exist.

\section{Results}

A total of 119 patients were recruited to the study. One centre failed to adhere to the trial protocol, and data for its 16 patients were excluded on the advice of the data monitoring committee (an audit of data indicated that control patients from the centre also received strategies identified as being part of the intervention). At baseline the intervention group (51 patients) and the control group (52 patients) were similar in terms of age, sex, diagnosis, and metastatic disease (table 1) and the outcome measurements for the groups did not differ significantly (table 2).

Sixteen patients died during the course of the study and 28 patients withdrew (table 3). Of the 27 patients who withdrew but did not report an improvement in their breathlessness, 16 withdrew because of a deterioration in their condition (13 control, 3 intervention, exact $\mathrm{P}=0.01$ ) and four were unhappy with the arm to which they had been allocated (3 control, 1 intervention). This left seven patients who withdrew for other reasons (2 control, 5 intervention). The major difference in the number of withdrawals between the groups therefore occurred where the patient's condition deteriorated. This was also reflected by the fact that the survival of the patients who withdrew from the control arm was significantly worse than the survival of patients withdrawing from the intervention arm (hazard ratio 2.5, $\mathrm{P}<0.05$, excluding the intervention patient who withdrew because he felt better). Survival of all withdrawals versus non-withdrawals was also significantly worse (hazard ratio 2.0, $\mathrm{P}<0.01$ ). All withdrawing patients or those who died were assumed to have a poor outcome relative to all the patients for whom an eight week assessment was available.

As overall survival of the two groups of patients did not differ significantly, it cannot be concluded that the intervention improved survival. However, the pattern of mortality showed that the intervention patients may have had improved survival over the first six months, but this was not maintained. Since the intervention under evaluation was non-pharmacological, it was important to establish whether the groups differed with regard to pharmacological intervention and if this could have accounted for differences observed in breathlessness scores. No appreciable differences in medication between the two groups were found. The proportion of patients taking opioids in intervention group patients at baseline was $22 \%$, and at 8 weeks $27 \%$, the corresponding figures for the control group were $23 \%$ and $33 \%$ (the respective percentages for other medications were: steroids-intervention 31\% and $45 \%$, control $27 \%$ and $30 \%$; bronchodilatorsintervention $27 \%$ and $27 \%$, control $31 \%$ and $44 \%$; non-opioid analgesics-intervention $51 \%$ and $33 \%$, control $44 \%$ and $55 \%$; antibiotics-intervention $5 \%$ and $15 \%$, control $2 \%$ and $11 \%$; and psychotropicsintervention $14 \%$ and $18 \%$, control $17 \%$ and $22 \%$ ).

At baseline both groups reported high levels of distress due to breathlessness and associated functional impairment (table 2). At 8 weeks, the intervention group showed significant improvement for breathlessness at best, WHO performance status, levels of depression, and physical symptom distress (table 4). Levels of anxiety and distress due to breathlessness improved slightly. The groups did not differ significantly in overall activity levels $(\mathrm{P}=0.10)$, but a secondary analysis of three specific subitems on the activity level scale (R41: climb stairs; R43: walk outdoors; and R44: go shopping) showed significant improvement in activity levels for the intervention group. (These three subitems had been chosen before the start of the analysis because they represented the main areas of activity that most participants rated as being problematic and because they were an objective measurement

Table 2 Baseline data for intervention and control groups

\begin{tabular}{lccccc} 
& \multicolumn{2}{c}{ Intervention group } & & \multicolumn{2}{c}{ Control group } \\
Questionnaire & $\begin{array}{c}\text { No of } \\
\text { patients }\end{array}$ & $\begin{array}{c}\text { Median } \\
\text { (range) }\end{array}$ & & $\begin{array}{c}\text { No of } \\
\text { patients }\end{array}$ & $\begin{array}{c}\text { Median } \\
\text { (range) }\end{array}$ \\
\hline Visual analogue scale: & & & & & \\
\hline Distress caused by breathlessness & 47 & $6(0-10)$ & & 49 & $5(0-10)$ \\
\hline Breathlessness at worst & 47 & $7.5(0-10)$ & & 49 & $7.9(0-10)$ \\
\hline Breathlessness at best & 47 & $4(0-9.1)$ & & 49 & $3.5(0-8.9)$ \\
\hline WH0 performance status & 49 & $2(0-3)$ & & 51 & $1(0-3)$ \\
\hline Hospital anxiety and depression scale: & & & & & \\
\hline Anxiety & 48 & $7(0-17)$ & & 49 & $6(0-17)$ \\
\hline Depression & 48 & $6(0-16)$ & & 49 & $5(2-14)$ \\
\hline Rotterdam symptom checklist: & & & & & \\
\hline Psychological symptoms & 48 & $14(7-27)$ & & 49 & $14(7-26)$ \\
\hline Physical symptoms & 48 & $50(34-77)$ & & 49 & $49(30-77)$ \\
\hline Activity (total items 38-44) & 45 & $12(7-26)$ & & 49 & $12(7-27)$ \\
\hline Activity (subitems R41, R43, R44) & 45 & $6(3-12)$ & & 49 & $5(3-12)$ \\
\hline Quality of life & 45 & $3(1-6)$ & & 49 & $3(1-6)$ \\
\hline
\end{tabular}

Scores of patients who died were not included in this analysis. In several cases data were missing because patients did not complete individual questions on the questionnaires.

Table 3 Reasons for patients' withdrawal after randomisation to study

\begin{tabular}{lcc} 
& Intervention group & $\begin{array}{c}\text { Control } \\
\text { group }\end{array}$ \\
\hline Progression of disease; too unwell to continue & 3 & 13 \\
\hline No explanation given & 2 & 1 \\
\hline Unhappy at being randomised to control group & 1 & 3 \\
\hline Unhappy at being randomised to intervention group & & 1 \\
\hline Developed new primary site requiring extensive surgery & 1 & \\
\hline Alcohol intoxication & 1 & \\
\hline Family commitments, bereavement & 1 & \\
\hline Did not like being questioned & 1 & \\
\hline Feeling better &
\end{tabular}


Table 4 Change between baseline and 8 weeks in intervention and control groups

\begin{tabular}{|c|c|c|c|c|c|}
\hline \multirow[b]{2}{*}{ Questionnaire } & \multicolumn{2}{|c|}{ Intervention group } & \multicolumn{2}{|c|}{ Control group } & \multirow[b]{2}{*}{$\begin{array}{c}P \\
\text { value }\end{array}$} \\
\hline & $\begin{array}{l}\text { No of } \\
\text { patients }\end{array}$ & $\begin{array}{l}\text { Median } \\
\text { (range) } \\
\text { change }\end{array}$ & $\begin{array}{l}\text { No of } \\
\text { patients }\end{array}$ & $\begin{array}{l}\text { Median } \\
\text { (range) } \\
\text { change }\end{array}$ & \\
\hline \multicolumn{6}{|l|}{ Visual analogue scales: } \\
\hline $\begin{array}{l}\text { Distress caused by } \\
\text { breathlessness }\end{array}$ & 49 & $0(-9-11)$ & 51 & $10(-7-11)$ & 0.09 \\
\hline Breathlessness at worst & 50 & $1(-7.2-8.5)$ & 52 & $4.8(-6.2-8.5)$ & 0.14 \\
\hline Breathlessness at best & 50 & $1.3(-7.1-8)$ & 52 & $7.0(-3.3-8)$ & 0.03 \\
\hline WHO performance status & 51 & $0(-3-3)$ & 52 & $2(-1-3)$ & 0.02 \\
\hline \multicolumn{6}{|l|}{ Hospital anxiety and depression: } \\
\hline Anxiety & 50 & $0(-7-11)$ & 52 & $9.5(-6-11)$ & 0.08 \\
\hline Depression & 50 & $0.5(-10-7)$ & 52 & $6(-7-7)$ & 0.02 \\
\hline \multicolumn{6}{|l|}{ Rotterdam symptom checklist: } \\
\hline Psychological symptoms & 50 & $1(-9-13)$ & 52 & $9(-8-13)$ & 0.21 \\
\hline Physical symptoms & 50 & $2.5(-24-16)$ & 52 & $14(-11-16)$ & 0.04 \\
\hline \multicolumn{6}{|l|}{ Activity: } \\
\hline Items 38-44 & 47 & $2(-12-15)$ & 52 & $8.5(-4-15)$ & 0.1 \\
\hline Subitems R41, R43, R44 & 47 & $0(-6-9)$ & 52 & $5.5(-3-9)$ & 0.05 \\
\hline Quality of life (1 item) & 47 & $1(-4-4)$ & 52 & $2(-2-4)$ & 0.25 \\
\hline
\end{tabular}

Negative scores show improvement.

of breathlessness comparable to the three subitems used for breathlessness in the European Organisation for Research and Treatment of Cancer's lung module. ${ }^{11}$ ) The groups were similar in breathlessness at worst, psychological distress, and overall global quality of life.

\section{Discussion}

Breathlessness in advanced lung cancer is an unpleasant and intractable problem that directly interferes with all aspects of daily living and can provoke intense anxiety. ${ }^{6}$ Patients may also receive little or no help or advice on how to cope during attacks of breathlessness. ${ }^{12}$ This is the first multicentre randomised controlled study that set out to evaluate nursing strategies for managing breathlessness in various treatment centres in the United Kingdom. The findings show that patients attending nursing clinics for breathlessness experienced improvements in breathlessness, performance status, and physical and emotional states.

Precisely how the intervention affects depression and anxiety is unclear. Changes from baseline to eight weeks in scores on the hospital anxiety and depression scale suggest a general improvement in mood for the intervention group. Two particular elements of the intervention might be responsible for the improvements (these had not been apparent in the pilot study): the emphasis on teaching more effective ways of coping with breathlessness and the opportunity to talk about difficult feelings and concerns.

\section{Possible criticisms}

The analysis rested on the assumption that patients who withdrew from the study had a poor outcome; clearly, it would have been preferable if their outcomes had actually been assessed. The method of analysis also assumed that all patients were able to show a change in either direction on the rating scales, but patients whose baseline measurements were at the extremes of a scale would be able to show change in only one direction. As the groups were similar at baseline, however, both groups should have been affected equally by this problem. Though the analysis of such a large number of
- In lung cancer, high levels of distress, anxiety, and functional impairment are associated with the symptom of breathlessness

- Evidence on the use of many treatments for this common and frightening symptom is lacking

- Interventions based on psychosocial support, breathing control, and learning coping strategies can help patients to cope with the symptom of breathlessness and reduce physical and emotional distress

outcomes would imply that one or two might be significant by chance even if the intervention had no effect, 5 out of 11 outcomes reached conventional levels of significance and all outcomes favoured the intervention group. Though the differences between the two groups were significant, the magnitude of the effect of intervention is more difficult to assess, and data need to be interpreted with caution. Not all patients benefited, but performance status gives an idea of the degree of benefit some patients experienced. The median change for the intervention group was 0: this group maintained the ability to carry out activities. For the control group there was a median deterioration of two points, so that for patients at baseline whose score was 2 (that is, up and about for $50 \%$ of waking hours, and capable of self care) typically deteriorated to grade 4 at eight weeks (that is, confined to bed or chair, no self care, completely disabled).

\section{Conclusion}

This study set out to evaluate a nursing intervention for breathlessness in patients with lung cancer and to replicate a previous study. ${ }^{6}$ The results confirm the findings from the earlier study and show that intervention based on psychosocial support, breathing control, and coping strategies can help patients deal with their breathlessness.

It was evident at the outset of the study that patients in both control and intervention groups experienced high levels of distress and functional impairment due to breathlessness. Most patients who managed to complete the study had a poor prognosis, and breathlessness was typically a symptom of their deteriorating condition. Considering the difficulties of randomising very ill patients to an eight week intervention study, the completion and results of this study are an achievement in the field of palliative care research. Multicentre trials of nursing intervention have not been reported, and we believed that this is the first study of its kind in the United Kingdom. The model of collaboration used between specialist nurses and the coordinating centre for the research may represent a useful approach to managing innovation within challenging areas of practice and may be of value for future studies of this nature.

We thank Macmillan Cancer Relief for funding this study, Professor Mike Richards and Dr Tim Sheard for advice on the conduct of the study, and the staff of the Clinical Trials Statistics Unit, Institute of Cancer Research, Sutton, Surrey, for providing an independent randomisation service. We especially thank and acknowledge the Macmillan and specialist nurses at the six par- 
ticipating centres: Sian Dennison, Tony Shute, and Glad Baldry, Plymouth Hospitals NHS Trust; Jo O'Neill, Neil Cliffe Cancer Care Charity, and Michael Connolly, Wythenshawe Hospital, Manchester; Diane Stidston, Anna Farrar, and Jane McKay, Norfolk and Norwich Healthcare NHS Trust; Rachel Hornsby, Cathy Penn, and Anne Noble, Southampton University Hospital Trust, Cancer Care Directorate; Brian Lowden and Norma Thomson, Hove General Hospital, Brighton Healthcare NHS Trust; Kay Doyle, Simon Jones, Tenovus Cancer Information Centre/ Llandough Hospital, Cardiff.

Contributors: JC had the original idea; initiated the study; developed the intervention; participated in establishing the study and supervising, and supporting researchers and nurses; and helped to interpret data. HP developed the intervention and participated in establishing the study and supervising and supporting researchers and nurses. $\mathrm{CB}$ developed the study protocol, intitated the study and training of nurses in each centre, and coordinated the study for the first half of data collection. $\mathrm{MB}$ coordinated the second half of the study and supported nurses in study sites; was responsible for data management, checking, and entry; and carried out data analysis. RA'H was statistical adviser to the project and helped with analysis and presentation of data. MK helped initiate the study, support researchers and nurses, and manage and check data. The Macmillan and specialist nurses listed above collected data. MB, JC and RA'H wrote the paper. All authors read and commented on the draft paper. JC and BM are guarantors.

Funding: Macmillan Cancer Relief.

Competing interests: Funding declared above.
1 O'Driscoll M, Corner J. The experience of breathlessness in lung cancer Eur J Cancer Care (in press).

2 Twycross RG, Lack SA. Therapeutics in terminal cancer. Edinburgh: Churchill Livingstone, 1986

3 Muers MF, Round CE. Palliation of symptoms in non-small cell lung cancer: a study by the Yorkshire Regional Cancer Organisation Thoracic Group. Thorax 1993;48:339-43.

4 Corner J, Plant H, Warner L. Developing a nursing approach to managing dyspnoea in lung cancer. Int J Palliative Nurs 1995;1:5-10.

5 Higginson I, McCarthy M. Measuring symptoms in terminal cancer: are pain and dyspnoea controlled? J R Soc Med 1989;82:264-7.

6 Corner J, Plant H, A'Hern R, Bailey C. Non-pharmacological intervention for breathlessness in lung cancer. Palliative Med 1996;10:299-305.

7 World Health Organisation. WHO handbook for reporting results of cancer treatment. Geneva: WHO, 1979. (WHO offset publication No 48.)

8 Zigmond AS, Snaith RP. The hospital anxiety and depression scale. Act Psychiat Scand 1979;67:361-70.

9 De Haes J, Olschewski M, Fayers P, Visser MRM, Cull A, Hopwood P, et al. The Rotterdam symptom checklist (RSCL): a manual. Groningen: Northern Centre for Healthcare Research, University of Groningen, 1996.

10 Gould AL A new approach to the analysis of clinical drug trials with withdrawals. Biometrics 1980;36:721-7.

11 Bergman B, Aaronson N K, Ahmedzai S, Kaasa S, Sullivan M for the European Organisation for Research and Treatment of Cancer (EORTC) Study Group on Quality of Life. The EORTC QLQ-LC13: a modular supplement to the EORTC core quality of life questionnaire (QLQ-30) for use in lung cancer clinical trials. Eur J Cancer 1994:30 A: 635-42.

12 Roberts D, Thorne SE, Person C. The experience of dyspnoea in late stage cancer: patients and nurses' perspectives. Cancer Nurs $1993 ; 16: 4: 310-20$

(Accepted 15 December 1998)

Cite this article as:

Bredin M, Corner J, Krishnasamy M, Plant H, Bailey C, A'Hern R. Multicentre randomised controlled trial of nursing intervention for breathlessness in patients with lung cancer [abdridged version]. BMJ 1999;318:901-4. (full version: www.bmj.com/cgi/content/full/318/ $7188 / 901)$ 